\title{
A GPRS-Based Low Energy Consumption Remote Terminal Unit for Aquaculture Water Quality Monitoring
}

\author{
Dan $\mathrm{Xu}^{1}$, Daoliang $\mathrm{Li}^{1,}{ }^{1,}$, Biaoqing $\mathrm{Fei}^{1}$, Yang Wang ${ }^{2}$, and Fa Peng ${ }^{3}$ \\ ${ }^{1}$ College of Information and Electrical Engineering, \\ China Agricultural University, Beijing 100083, China \\ ${ }^{2}$ College of Engineering, China Agricultural University, Beijing 100083, China \\ ${ }^{3}$ College of Mechanical and Electronic Engineering, \\ Shangdong Agricultural University, Taian 271000, China \\ dliangl@cau.edu.cn
}

\begin{abstract}
The monitoring of water quality parameters such as DO, pH, salinity and temperature are necessary for the health of seafood such as sea cucumber. However, traditional monitoring system is based on cable data acquisition that has many disadvantages. Nowadays, GPRS is the most commonly accepted way for wireless transmission. Based on it, a type of low energy consumption RTU is developed and applied. In this paper, details of the design are introduced. In hardware design of this type of RTU, STM8L152 is selected in the MCU module to accomplish the function of ultralow power consumption, and solar battery is designed to solve the problem of power supply. In software design of the RTU, the sleep/online mode conversion is programed to reduce the energy consumption. It is comparatively low-priced and can detect necessary parameters for aquaculture. Performances of the RTU are tested in experimental stations and compared with two advanced water quality analyzers. Results show that it shows almost the same variation characteristics as those of $\mathrm{HACH}$ and YSI.
\end{abstract}

Keywords: RTU, GPRS, aquaculture, low energy consumption.

\section{Introduction}

Aquaculture is a fast growing food-producing part in the world. [1] In China, there are lots of problems in traditional aquaculture, such as pollution and consumption. [2] In modern aquaculture management, it's most important to monitor water quality reliably and control water environment in time. It's useful in enhancing the fish concentration and growth rate, at the same time reducing the occurrence of fish diseases. Dioxide oxygen (DO), $\mathrm{pH}$, salinity and temperature are most important factors in sea water quality for aquaculture. In order to improve the level of aquaculture, these parameters must be precisely monitored. [3]

Traditional monitoring system is based on cable data acquisition that has more and more disadvantages. [4] In the process of wiring cable technology, there are a series 
of restrictions, such as high temperature, high pressure, high altitude and high risks. First, it is difficult for the installation of data acquisition devices, which will increase the maintenance workload. Second, quantities of cables will have a bad impact on visual appeal and increase the cost. Third, cables face the challenges of aging and the risk of bitten by rodents and other animals, which leads to a rise of fault rate. To tackle these issues, wireless data acquisition is adopted.

Within the past decade, large numbers of water quality monitoring instruments have been commercialized. Some integrated with remote real-time water quality monitoring systems have been developed and deployed by scientists, governmental agencies and industries throughout the world in modern aquaculture. [5] Nowadays two wireless ways are mainly introduced, wireless sensor network (WSN) and General Packet Radio Service (GPRS). [6] Wireless sensor network is a kind of wireless network without infrastructure. [7] It receives and sends messages through wireless and self-organization multi-hop routing. [8] It is a relatively new wireless technology that has sharp technical problems that is instability. On contrast, GPRS is a relatively mature technology that has been adopted widely in daily life, such as mobile office, mobile commerce, mobile information service, mobile internet and multimedia business. [9]

GPRS is a new type of data transmission technology based on Global System of Mobile Communication (GSM). It adopts packet switching mode, which occupy wireless resources only in the process of sending and receiving data. In theory, it can reach transmission data rate as high as $171.2 \mathrm{Kbit} / \mathrm{s}$. Except for the advantage in speed, it is also always on-line. That means users can keep contact with the net at any time. [10]

Based on the advantages of GPRS, a new type of remote terminal unit (RTU) is developed and applied in the experiment stations. It detects $\mathrm{DO}, \mathrm{pH}$, salinity and temperature at the same time.

In this paper, details of design of this type of RTU are introduced. And tests of working performances about its detecting parameters are done and analyzed. Finally we reach a conclusion about application of this RTU.

\section{Materials and Methods}

\subsection{Selection of Monitoring Sites}

To test the performances of this type of RTU, two experimental stations in the city of Weihai in Shandong Province are established. One is in Wendeng Ocean and Fishery Bureau, another is in Shandong Xunshan Aquatic Product Group Corporation.

The city of Weihai (N 36 $41^{\prime} \sim 37^{\circ} 35^{\prime}$, E $121^{\circ} 11^{\prime} \sim 122^{\circ} 42^{\prime}$ ) locates in the eastern most part of Shandong peninsula. With three sides (east, south, north) facing the sea and a coastline of $985.9 \mathrm{~km}$, it's also sees the Korean peninsula and the Japanese islands across the sea. [11] It is rich in marine resources. And the market driven stimulus keeps mariculture a rapid growth year by year. [12] However, the science and innovation level is still low. Extensive pattern remains on the development and utilization of marine resources. Many problems still exist, such as the great need of 
professionals, the lack of high-tech research results, and the low level and slow pace in the industrialization of the project.

Based on those advantages and places need improvement, high-tech research results are integrated into this area and two experimental stations in the city of Weihai are built. The two experimental stations aim mainly at raising sea cucumber and abalone. Sea cucumber and abalone are both traditional Chinese seafood with high edible and medicinal value. In recent years, due to the worldwide over-development of sea cucumber resources and the sharp decline of its population, sea cucumber artificial breeding is springing up. [13] However, these precious sea creatures also demands strict living conditions. DO, PH, salinity and temperature have to be precisely detected and controlled in a defined range. For example, sea cucumber is cold temperature zone species. Its living water temperature is $-1.5 \sim 30^{\circ} \mathrm{C}$. It takes in less food and be in the process of half dormancy when the water temperature is below $3^{\circ} \mathrm{C}$. It reaches the highest food consumption when the water temperature is $10 \sim 15^{\circ} \mathrm{C}$. Then food consumption falls sharply when it is $10 \sim 15^{\circ} \mathrm{C}$. Then it goes into aestivation when it is over $20^{\circ} \mathrm{C}$. So, the most moderate water temperature is $3 \sim 20^{\circ} \mathrm{C}$. And it stops growing when it is below $2^{\circ} \mathrm{C}$ and over $23^{\circ} \mathrm{C}$. [14] Thus, large numbers of RTUs are installed which can detect these parameters in the two experimental stations.

From July 25th to July 28th in the year of 2013, performances of the RTUs installed in the two experimental stations are tested. Large numbers of experiments are done and useful data is collected for future analysis. Lots of problems are found out. Most problems are solved, while some problems are leaving unresolved but already reported for further study.

\subsection{Hardware Design}

This type of RTU consists mainly of 4 modules. They are sensor module, MCU module, GPRS module and power module. Their relations are shown in figure 1.

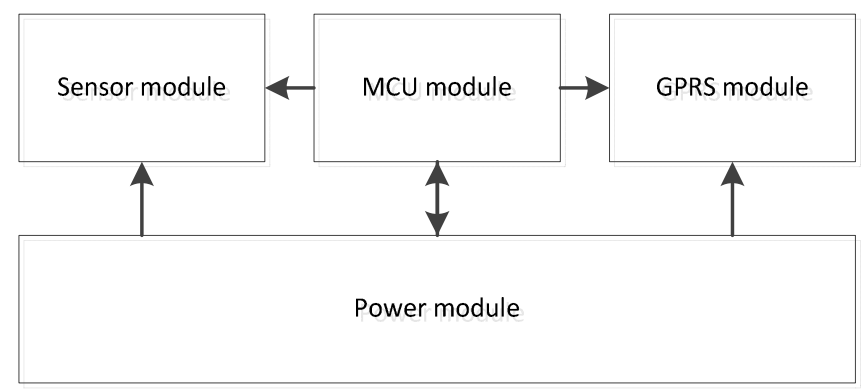

Fig. 1. Relations between different modules

The sensor module can detect necessary parameters of water quality. It consists of DO sensor, $\mathrm{pH}$ sensor, salinity sensor and temperature sensor. The MCU module processes the data collected by sensors, then sends the data to GPRS module. The GPRS module sends the processed data to the monitoring center. The power module is controlled by the MCU module and supply power to the other three modules. 
After PCB plate-making and circuit debugging, hardware performs well.

Then details about the system are shown as follows.

\subsubsection{Sensor Module}

The sensor module is important part of the RTU. It detects on-line necessary water quality parameters, including $\mathrm{DO}, \mathrm{pH}$, salinity and temperature. The temperature sensor is attached to DO sensor electrode. So there are altogether three electrodes. Sensors send data about water quality to MCU through the signal wires, at the same time suspending in the water depending on signal wires.

4-core water-proof cables are selected as signal wires. They connect sensors and MCU. They have three functions. The first function is to supply power to the sensors. The second function is to send data collected by sensors to MCU. The third function is to act as suspension wire to suspend sensors in the water. The lengths of signal wires are adjustable so that sensors can detect water quality information in different depths according to different application requirements.

\subsubsection{MCU Module}

The MCU module is core part of the RTU. It has four functions. First, it controls the power module that supplies power to each module. Second, it controls sensors to collect data. Third, it controls the GPRS module to send the data wirelessly. Last, it processes all the data. To cut down the cost and energy consumption, STM8L152 is selected in the MCU module. [15] It is an ultra-low power consumption MCU chip of ST Corporation. And it has the following advantages. [16]

First, it has a wide working voltage range: from $1.8 \mathrm{~V}$ to $3.6 \mathrm{~V}$ or form $1.65 \mathrm{~V}$ to $3.6 \mathrm{~V}$. (Its minimum working voltage is $1.65 \mathrm{~V}$ in the mode of power-down).

Second, it has five ultralow energy consumption modes: low power running mode $(5.1 \mu \mathrm{A})$, low power waiting mode $(3.0 \mu \mathrm{A})$, real time clock operation suspend mode $(1.2 \mu \mathrm{A})$, self-wakeup suspend mode $(0.91 \mu \mathrm{A})$ and SRAM content retaining suspend mode $(350 \mathrm{nA})$. They play a key role in cutting down the energy consumption of the whole system.

Third, its operating temperature range is from $-40^{\circ} \mathrm{C}$ to $85^{\circ} \mathrm{C}$, which enables it working under different severe environments.

Forth, it has rich internal functions. It integrates SPI function internally which makes it convenient for the operation of wireless communication. And it integrates EEPROM and LCD function to facilitate the expansion of the function.

Fifth, compared with MSP430 series MCU, STM8L series MCU has lower prices and higher cost performance.

\subsubsection{GPRS Module}

The core chip of the GPRS module is SIM900. [17] It is an ultra compact and reliable wireless module presented by the corporation of SIMCom. It is a complete Quad-band GSM/GPRS module in a SMT type and designed with a very powerful single-chip processor integrating AMR926EJ-S core, allowing you to benefit from small dimensions and cost-effective solutions. 
Featuring an industry-standard interface, the SIM900 delivers GSM/GPRS 850/900/1800/1900MHz performance for voice, SMS, Data, and Fax in a small form factor and with low power consumption. With a tiny configuration of $24 \mathrm{~mm} \times 24 \mathrm{~mm} \times 3 \mathrm{~mm}$, SIM900 can fit almost all the space requirements in your M2M applications, especially for slim and compact demands of design.

\subsubsection{Power Module}

The power module consists of solar panel, charging control module and storage battery. The charging control module converts solar energy collected by the solar panel directly into electrical energy and stores it in the storage battery. The storage battery supplies power to the whole system.

The core chip of the power module is CN3063. [18] CN3603 is a single-cell lithium battery charge management chip that can be used in the solar power supply. Thermal modulation circuit can control the chip temperature in a safe range when in face of high power consumption of the device or high ambient temperature. Charging current is set through an external resistor. When the input voltage is powered down, CN3063 enters the mode of low-power sleep automatically. At this time the current consumption of the battery is less than $3 \mu \mathrm{A}$. It also involves other functions like lockout of low voltage input, automatic recharge, battery temperature monitoring and indicators of charging states and end of charging states.

\subsubsection{Operating Principle}

In the RTU system, the input end of charging control module connects with the solar panel. The output end of charging control module connects with the storage battery. The storage battery connects with the MCU power input end. The power output end of MCU connects with charging control module, sensors and GPRS module. The charging control module, storage battery, MCU and GPRS module are placed in the circuit box. Among them, MCU module and GPRS module are integrated in the main circuit board. Antenna is settled on the main circuit board to send data. There are four sensor interfaces on the bottom of circuit. Signal wires of sensors connect with circuits in the circuit box through these interfaces. RS485 is adopted as the communication interface. There are also fixing holes on the bottom of circuit box. Ribbons can pass through these fixing holes to tie the RTU to the support rod above the water surface. Solar panel is fixed on top of the circuit box to collect solar energy. The 4-core waterproof cables act as signal wires. They connect to sensors to one end, and connect to MCU in the circuit box through waterproof interfaces. The lengths of signal wires are adjustable so that sensors can be placed in different level in the water.

\subsection{Software Design}

\subsubsection{Energy Consumption Reducing Software}

This system works in two modes, they are online and sleep. It is necessary for reducing the energy consumption. When the system is not on the process of collecting 
or processing data, it is set on sleep mode. When the water quality parameters need to be detected, it turns to online mode automatically to implement the data collecting, processing and sending. The intervals of sleep/online mode changing are previously integrated in the MCU. The intervals of online detecting can be the same. Every few hours, intervals of detecting can be changed according to values of parameters. For example, DO can be detected more intensely at the time before down when it is low.

\subsubsection{Operating Principle}

The system sets a sampling interval through programming. Sensors collect data once among each interval. The MCU supplies power to sensors only on the process of data sampling, while cuts off the power at other time, so as to cut down the energy consumption. The data collected by sensors is sent to MCU through signal wires for processing. The processed data is sent through GPRS to the monitoring center.

The flow chart of main program is shown in figure 2.

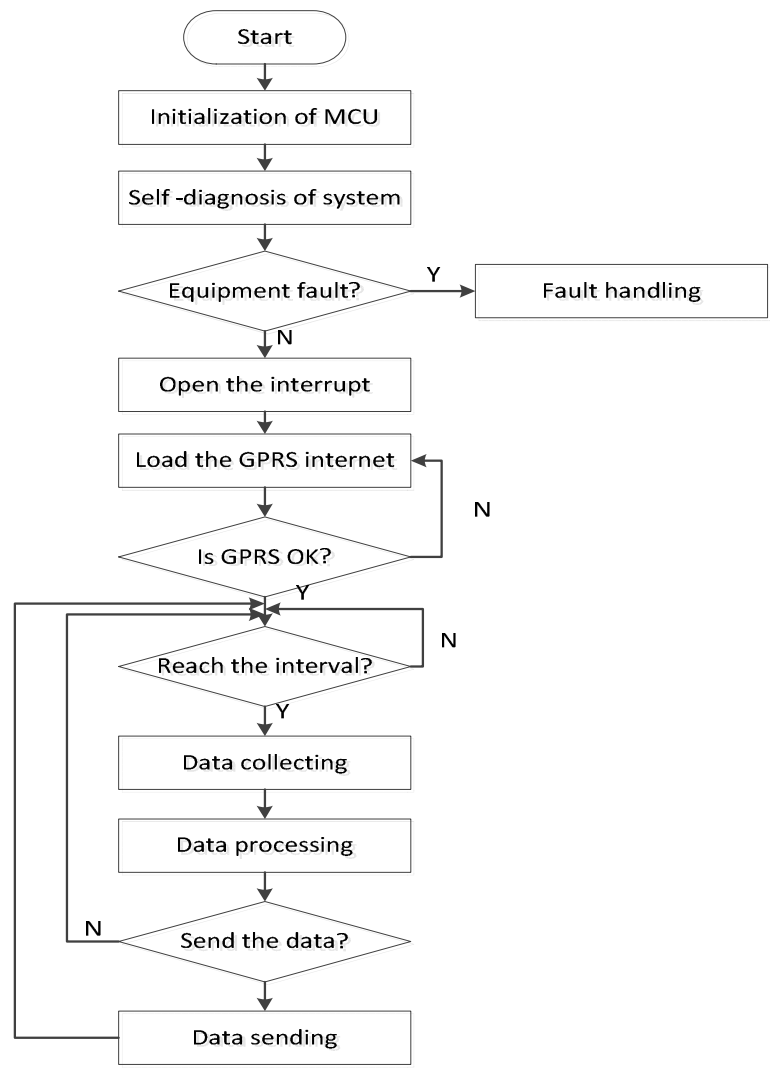

Fig. 2. The flow chart of main program 
Charts of subprograms are shown in figure 3.

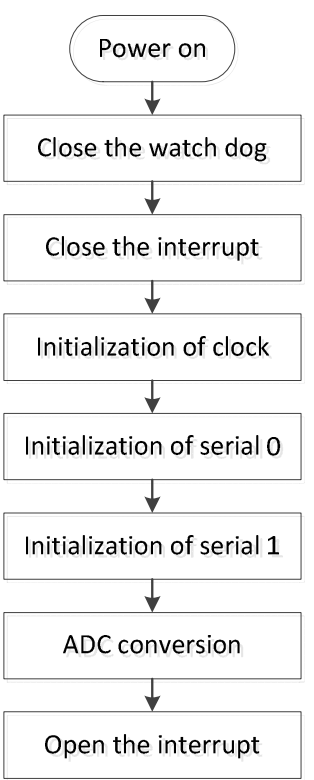

Fig. 3.1 Initialization

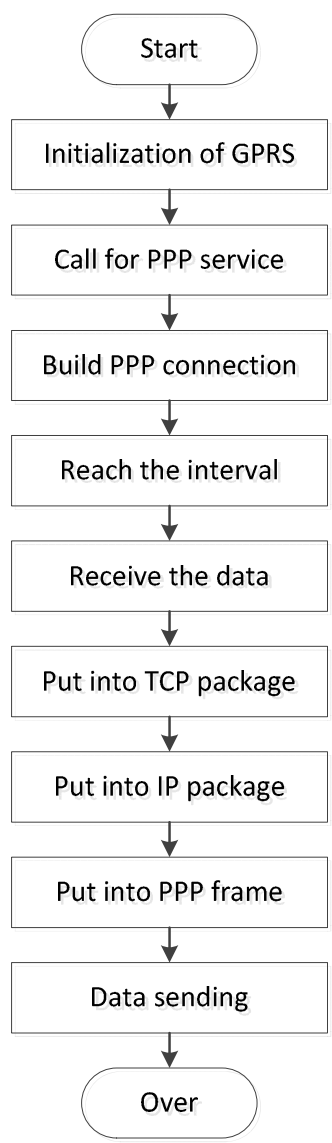

Fig. 3.2 GPRS data transmission

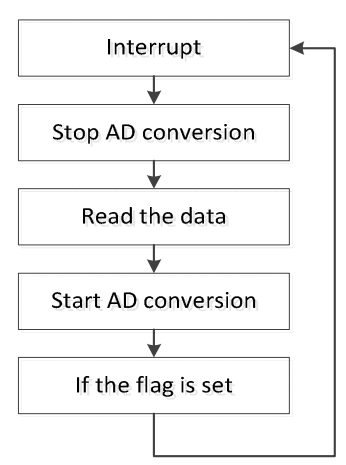

Fig. 3.3 Analog data acquisition

\section{Results and Discussion}

\subsection{Experiments}

From July 25th to July 28th in the year of 2013, performances of the RTUs installed in the two experimental stations are tested. Large numbers of experiments are done and useful data is collected for the future analysis. From 12:30 to 17:15 of each day, $\mathrm{DO}, \mathrm{pH}$, salinity and temperature in these experiment stations are collected by three different instruments for comparison. They are RTU, HACH and YSI.

$\mathrm{HACH}$ helps water resource professionals generate reliable data throughout the entire cycle of water, from measuring precipitation to monitoring estuaries and 
ground water, and everything in between. Hydrolab multi-parameter water quality instruments are built using the industry's leading sensor technology. [19]

YSI has been used for many years in facilities that process wastewater generated by metal finishing plants, but recently it has become prominent in municipal wastewater treatment plants. [20] The probe contains a sensor that measures electrical charges from particles, called ions, and these charges are converted to millivolts $(\mathrm{mV})$ that can be either negatively or positively charged. And like all sampling measurements taken by operators, they are snapshots in time that can indicate process efficiency and identify treatment problems before they affect effluent quality. When using continuous monitoring and control instrumentation, this snapshot can become a real-time indicator.

$\mathrm{HACH}$ and YSI are all advanced water quality monitoring instruments produced in America that can detect precise water quality parameters respectively. To have a contrast with the performances of RTU, useful data is collected from time to time in different test points by these three instruments.

\subsection{Results}

DO values collected by these instruments from time to time are shown in figure 4 . (Data is collected in July 28th)

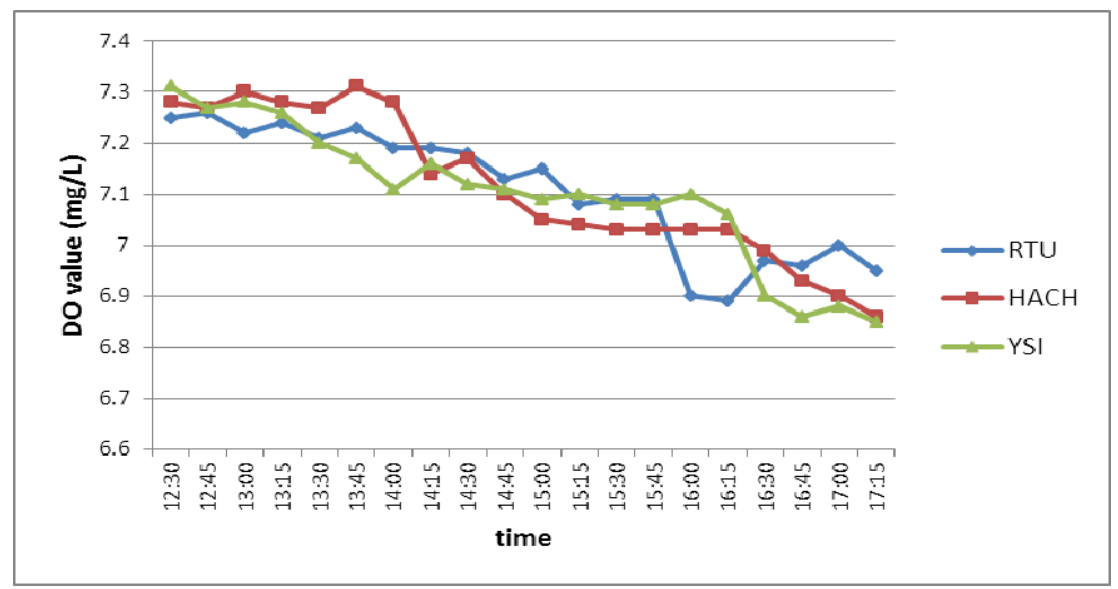

Fig. 4. DO values collected by three instruments

$\mathrm{PH}$ values collected by these instruments from time to time are shown in figure 5 . (Data is collected in July 27th) 


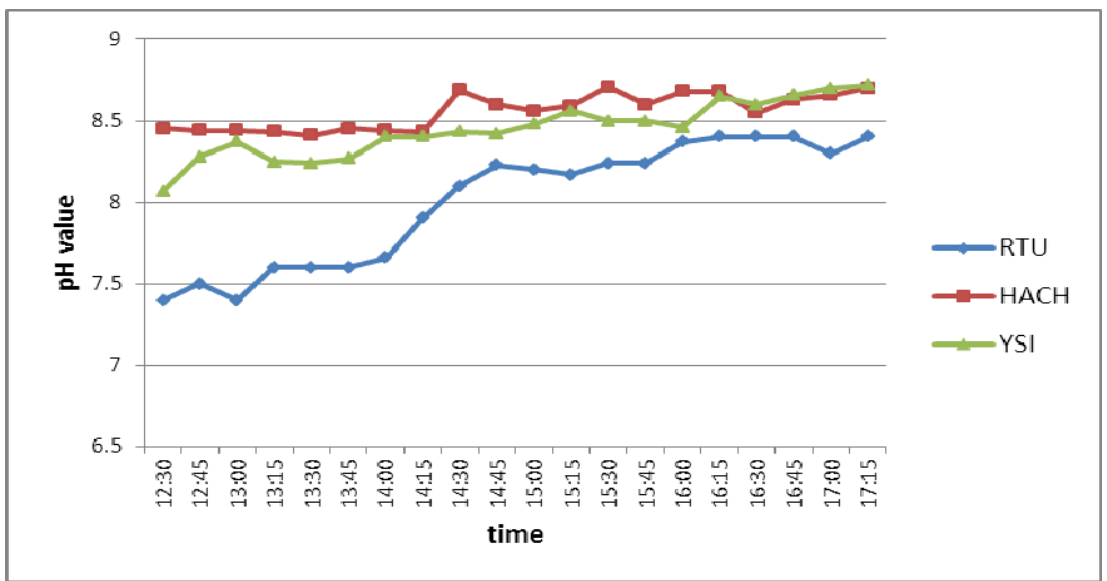

Fig. 5. PH values collected by three instruments

Salinity values collected by these instruments from time to time are shown in figure 6. (Data is collected in July 26th)

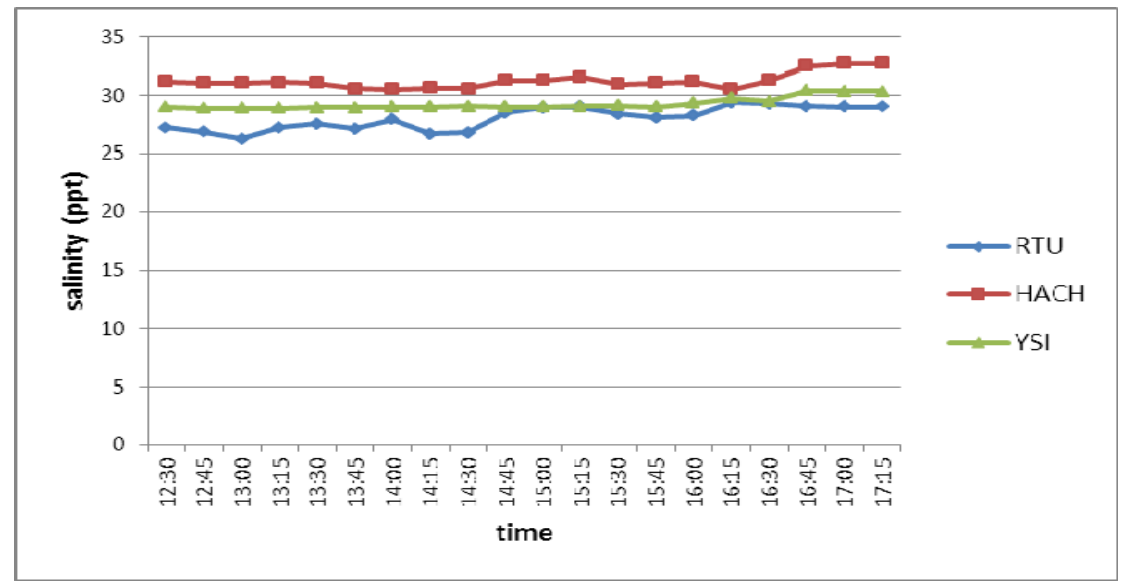

Fig. 6. Salinity values collected by three instruments

Temperature values collected by these instruments from time to time are shown in figure 7. (Data is collected in July 25th) 


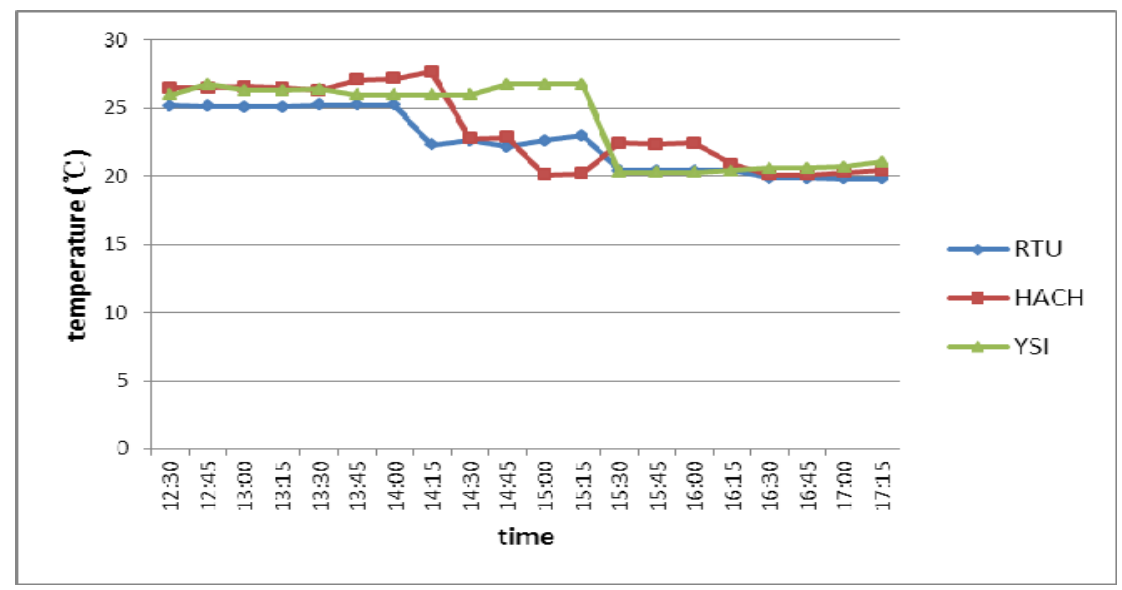

Fig. 7. Temperature values collected by three instruments

As these figures show, parameters collected by RTUs are almost the same precision as the other two famous instruments. It means that this type of RTU is in good performances.

Abnormal data may be caused by different environmental problems. Such as the instability of sensors, failure of power, errors in operation, and so on.

\subsection{Discussion}

Data collected by HACH and YSI shows the variation characteristics of these four parameters. From 12:30 to 17:15 of each day, DO, pH, salinity and temperature show different variation characteristics. DO decreases over time during this period. While $\mathrm{pH}$ increases over time during this period. Salinity has low fluctuations but it increases as the sea water evaporates and it reaches its peak at about 17:00. Temperature reaches its peak at about 14:00 and then it decreases as time goes. Data collected by RTU shows almost the same variation characteristics as those of HACH and YSI. Though not stable sometimes, it shows comparative good performances on the whole.

Though RTUs in most test points perform well, data collected in some test points of these experiment stations is abnormal. But in fact it has nothing to do with the RTU itself. It is sensors that are not stable. For example, some DO values detected by sensors are normal in the first couple of days. But they turn abnormal after about 14 or 15 days. So the DO sensors will have to be cleaned in about 10 days or so to gain accurate values. It is very inconvenient. So the urgent affairs should be the independent development of more stable and more accurate sensors. 


\section{Conclusion}

(1) This study researches into a new type of RTU based on GPRS. It is applied in some aquaculture experiment stations. It provides a new technical solution for improving the backward breeding way and aquaculture environment monitoring method of the Chinese aquaculture field.

(2) In hardware design, STM8L152 is selected in the MCU module to accomplish the function of ultralow power consumption, and solar battery is designed to solve the problem of power supply. STM8L152 is comparatively low-priced, thus it cuts down the cost.

(3) In software design, the sleep/online mode conversion is programed to reduce the energy consumption.

(4) To test performances of this RTU, large numbers of experiments are done and useful data is collected in the experiment stations for further study. From the data analysis, we can see that it shows almost the same water quality parameters variation characteristics as those of HACH and YSI. Though not stable sometimes, it shows comparative good performances on the whole.

Acknowledgements. This work was supported by the National Agricultural Science and technology achievement transformation project (2012GB2E000330), the state of 12th five-year science and technology support projects (2012BAD35B03) and (2011BAD21B01), and the programs "Agro-scientific Research in the Public Interest" (201203017).

\section{References}

1. Hongbin, L., Guoxiang, H., Dakui, F., Bangding, X., Lirong, S., Yongding, L.: Prediction and elucidation of the population dynamics of Microcystis spp. in Lake Dianchi (China) by means of artificial neural networks. Ecological Informatics, 184-192 (2007)

2. Marcella, B., Wolf, A.: Planning for sustainable aquaculture. Tilapia Farming in the United States, China and Honduras, 1-39 (2013)

3. Liu, A., Junsheng, G., Meiyan, W.: Effects of Nanomaterials on Water Quality of Aquiculture. In: 2013 Third International Conference on Intelligent System Design and Engineering Applications, pp. 688-691 (2013)

4. Mingyou, Q., Lei, H., Wensheng, G.: Design and Realization of a Spot Data Acquisition System Based on ZigBee. Telecommunication Engineering, 34-38 (2008)

5. Ma, D., Ding, Q., Li, Z., Li, D., Wei, Y.: Prototype of an Aquacultural Information System Based on Internet of Things E-Nose. Intelligent Automation \& Soft Computing, 569-579 (2012)

6. Yuwen, S., Mingxia, S., Yingjun, X., Mingzhou, L., Longshen, L., Xiaoli, K.: Design on the System of Collection, Storage and Release for Field Information. Advanced Materials Research, 347-353 (2011)

7. Jingtao, W., Changbao, M., Yongbo, L.: Design of Wireless Sensor Network Node for Water Quality Monitoring. Computer Measurement \& Control, 2575-2578 (2009) 
8. Zhiguo, D., Deqin, X., Yunhua, Z., Guozhen, O.: Design of Water Quality Monitoring Wireless Sensor Network System Based on Wireless Sensor. Computing Engineering and Design, 55 (2008)

9. Xiaoyan, Z., Yong, X., Tingbo, J., Jinhua, W., Hongwu, H.: Research on the PowerFrequency Electric Field Measuring System Based on GPRS. Applied Mechanics and Materials, 246-247 (2012)

10. Jianxiang, L., Chongguang, F., Tao, L., Haibo, L.: Design of Electric Vehicle Data Acquisition and Transmission System Based on GPRS and Zigbee. In: Proceedings of the 2012 Second International Conference on Electric Information and Control Engineering, pp. 542-545 (2012)

11. Song, P.: Thought and Suggestions about Development of Blue Economy in the City of Weihai. Northern Economy, 70-72 (2011)

12. Sun, Z., Shuchao, L.: "Blue and Yellow" Strategic Perspective Transformation and Development of Mariculture. Shandong Social Sciences, 144-148 (2012)

13. Min, S., Ji, C., Daoliang, L.: Water temperature prediction in sea cucumber aquaculture ponds by RBF neural network model. In: 2012 International Conference on Systems and Informatics, pp. 1154-1159 (2012)

14. Ji, C., Li, D., Du, S., Wei, Y., Tai, H.: A Wireless Sensor Network Based Water Temperature Stratification Monitoring System for Aquaculture of Sea Cucumber. Sensor Letters, 1094-1100 (2011)

15. Li, S.: Study on Vehicle Detection Technology Based on AMR Sensor. Journal of Hubei University of Education, 48-50 (2012)

16. Zheng, G., Xu, Z., Qiu, Y.: Design of a Low Energy Consumption Wireless Sensor. Fujian Computer, 39-40 (2012)

17. Jay, M., Shah, S.: Simplified Secure Wireless Railway for Public Transport. In: 2013 Fifth International Conference on Computational Intelligence, Communication Systems and Networks, pp. 77-82 (2013)

18. Ianmin, H., Gao, Y.: Greenhouse wireless sensor network monitoring system design based on solar energy. In: International Conference on Challenges in Environmental Science and Computer Engineering, pp. 475-479 (2010)

19. Chen, G.: Detecting total phosphate in water with the instrument HACH. Industrial Water Treatment, 62-63 (2010)

20. Kaishan, S., Lin, L., Tedesco, L., Clercin, N., Hall, B., Shuai, L., Kun, S., Dawei, L., Ying, S.: Remote estimation of phycocyanin (PC) for inland waters coupled with YSI PC fluorescence probe. Environmental Science and Pollution Research, 5330-5340 (2013) 